Research Paper

\title{
Management of Inguinal Involvement of Peritoneal Surface Malignancies by Cytoreduction and HIPEC with Inguinal Perfusion
}

\author{
Yair Shachar1, Mohamed Adileh¹, Assaf Keidar1, Luminita Eid², Ayalah Hubert³, Mark Temper ${ }^{3}$, Salah \\ Avital $^{6}$, Aviram Nissan ${ }^{1 凶}$ \\ 1. The Department of Surgery, Hadassah Hebrew University Medical Center, Ein Kerem, Jerusalem, Israel; \\ 2. The Department of Anesthesiology, Hadassah Hebrew University Medical Center, Ein Kerem, Jerusalem, Israel; \\ 3. The Department of Oncology, Hadassah Hebrew University Medical Center, Ein Kerem, Jerusalem, Israel; \\ 4. The Department of Oncology, Rambam Medical Center, Haifa, Israel; \\ 5. The Department of Oncology, Sha'arei Tzedek, Medical Center, Jerusalem, Israel; \\ 6. Bon Secours Cancer Institute, Richmond, VA, USA.
}

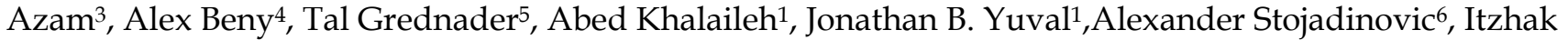

$\square$ Corresponding author: Aviram Nissan, M.D., Head, Department of Surgery, Hadassah Hebrew University Medical Center, Ein Kerem, POB 12000, Kyriat Hadassah, Jerusalem, Israel 91120. Telephone: +972-2-6779500; Fax: +972-2-6779510; e-mail: anissan@hadassah.org.il

(C) 2015 Ivyspring International Publisher. Reproduction is permitted for personal, noncommercial use, provided that the article is in whole, unmodified, and properly cited. See http://ivyspring.com/terms for terms and conditions.

Received: 2014.08.13; Accepted: 2014.09.26; Published: 2015.01.18

\begin{abstract}
Background: Achieving complete cytoreduction of peritoneal surface malignancies (PSM) can be challenging. In most cases, delivery of heated intra-peritoneal chemotherapy (HIPEC) is straightforward. However, using the closed technique in some cases may be technically challenging; for example, in patients requiring abdominal closure using a large synthetic mesh. In cases where groin hernias are present, it is imperative to resect the hernia sac, since it may contain tumor deposits. In cases with major inguinal involvement where disease may spread out of the hernia sac or in cases where a hernia repair was performed while disease is present, inguinal perfusion should be considered.

Aim: To describe our experience with combined intra-peritoneal and inguinal perfusion of HIPEC following cytoreductive surgery.

Patients and Methods: This is a retrospective review of all patients who underwent cytoreductive surgery (CRS) and HIPEC at our institution. A prospectively maintained database containing data of patients treated by CRS and HIPEC $(n=122)$ was reviewed. All patients with macroscopic inguinal involvement by PSM with complete cytoreduction perfused by HIPEC were included.

Results: We identified five cases who underwent CRS and combined intraperitoneal and inguinal perfusion after resection of large inguinal tumor deposits $(n=4)$ or after a recent hernia repair with hernial sac involvement by mucinous adenocarcinoma $(n=1)$. All five patients were successfully perfused using an additional outflow catheter placed in the groin.

Discussion: In cases of inguinal involvement by PSM, complete cytoreduction should be achieved and perfusion of the involved groin considered as it is feasible and safe.
\end{abstract}

Key words: miR-21, recurrence, prostate cancer

\section{Introduction}

Treatment of peritoneal surface malignancies (PSM) by cytoreductive surgery (CRS) combined with heated intraperitoneal chemotherapy (HIPEC) is an evolving technique with encouraging results [1]. The 
peritonectomy procedures are conducted according to the guidelines described by Sugarbaker [2]. Patient selection for the procedure requires a multi-disciplinary approach and combines many clinical as well as pathological variables.

The absence of extra-abdominal disease and the ability of a patient to undergo such a complex procedure are initially evaluated ${ }^{i}$. In patients with good performance status, the extent of disease, as reflected by the Peritoneal Cancer Index (PCI), tumor histology and response to prior systemic therapy are all taken into consideration of patient selection for CRS and HIPEC [3] [4] [5]. In most cases, extent of disease spread in the peritoneum is under-estimated by cross-sectional imaging. Therefore, in selected cases, staging laparoscopy may be used for determination of resectability [6].

The presence of PSM in the groin may be related to pre-existing inguinal hernia or in the cases of carcinoma of the ovary - regional lymphatic spread. In a large series of 217 patients with PMP described by Esquivel et al [7], 14\% of the patients presented with new-onset hernias, the majority of which were inguinal. Tumor deposits in the inguinal canal may be detected by cross sectional imaging as well as at time of inguinal surgery, and should be considered in surgical planning [7-12]. In both cases complete excision is warranted. However, perfusion of the inguinal canal during HIPEC should be considered, as it is readily achieved in a safe and effective manner. We propose a simple method practiced by our team for inclusion of the inguinal canal in HIPEC.

\section{Patients and Methods}

The multi-disciplinary approach for the treatment of PSM in our institution was established in 2007. A dedicated team was selected and trained in high-volume centers in the USA and Germany. Each patient is initially evaluated at the Surgical Oncology Clinic. Patients with good performance status and without extra-peritoneal disease are presented in a multi-disciplinary conference (Tumor Board). All cross sectional imaging and histopathology are reviewed and a treatment plan is agreed-upon by subject matter experts.

Selection criteria are in accordance with the PSOG guidelines [13] and the Milan conference [14]. Patients with limited liver metastasis are considered for CRS+HIPEC as well as patients with direct invasion of tumor into the pelvic side wall, groin or retroperitoneum. Patients with retroperitoneal lymph nodes involved by disease are excluded as well patients with disease progressing under systemic therapy and patients with metastasis to organs other than the liver, or with extensive liver involvement by tumor.

All procedures were performed under combined general endotracheal and epidural anesthesia in the lithotomy position. Cytoreduction was performed by the technique described by Sugarbaker [2] with some modifications.

HIPEC was delivered by the "closed" technique using a roller pump HT-1000 (Theramsolutions ${ }^{\mathrm{TM}}$, cases \#1-\#60) and a rapid infusion pump (Belmont ${ }^{\mathrm{TM}}$, Boston, USA, cases \#61-\#122). A single inflow catheter is placed on the liver surface with three outflow catheters placed in the right and left sub-phrenic spaces and in the pelvis. Inflow temperature is set for $44^{\circ} \mathrm{C}$. Outflow temperature is measured using a pelvic thermometer, tissue temperature is measured using a thermometer coupled to the Foley catheter. Core temperature is measured using an esophageal temperature probe. Flow and intra-abdominal pressure are constantly measured and closely monitored throughout the HIPEC procedure.

Selection of cytotoxic agents for HIPEC is based on previously reported protocols adopted by our group, with modifications according to patient's age, renal function and previous systemic chemotherapy used before the HIPEC procedure.

\section{Data capture}

For each patient scheduled to undergo CRS+HIPEC, a staging form is included in the chart including pre-operative PCI (as measured by cross-sectional imaging), intra-operative PCI, post-CRS-PCI, completeness of cytoreduction (CC-score), and each peritonectomy procedure and organ resection performed, as well as the agents delivered and the duration of the HIPEC procedure.

The aforementioned data, along with the operative report, discharge summary and pathology report are entered by a database manager into a computerized database in real-time fashion. Each follow-up visit to the clinic is recorded in the electronic medical record and the status of the patient is updated in the database.

\section{Results}

Between 2007 and 2013, there were 122 patients eligible for CRS + HIPEC. Complete cytoreduction was achieved in 101 (83\%). All patients with complete cytoreduction (CC0-1) underwent peritoneal perfusion. All, but one patient, were perfused in the operating room. One patient was not hemodynamically stable at the end of the procedure and therefore underwent perfusion on the first post-operative day in the intensive care unit. 
Table 1: Patients' characteristics

\begin{tabular}{|c|c|c|c|c|c|c|c|c|}
\hline Patient & Age & Sex & pathology & Pre-operative PCI & Intra-operative PCI & CC-score & Peri-operative complications & Discharge POD \\
\hline A & 65 & $\mathrm{~m}$ & PMP & 16 & 26 & 0 & None & 15 \\
\hline B & 53 & $\mathrm{f}$ & Ovary & 3 & 5 & 0 & Acute renal failure & 15 \\
\hline C & 54 & $\mathrm{f}$ & Ovary & 9 & 3 & 0 & Infected groin seroma & 26 \\
\hline $\mathrm{D}$ & 77 & $\mathrm{f}$ & PMP & 25 & 28 & 0 & Pulmonary embolism & 32 \\
\hline E & 70 & $\mathrm{~m}$ & PMP & 39 & 31 & 1 & Pneumonia & 19 \\
\hline
\end{tabular}

In five patients inguinal involvement was identified and completely resected (Table 1). The groin, in all five patients, was successfully perfused by inserting a fourth outflow catheter into the inguinal region, thereby directing the heated chemotherapy into the groin. Four out of five patients had minor peri-operative complications (Table 1). Patient $C$ suffered a grade II infected seroma at her groin, which may be attributed to the groin perfusion. All complications improved with conservative treatment, and all patients were discharged from the hospital in good condition. Average post-operative day at discharge was 21.4 days. None of the patients required a second operation during the initial hospitalization.

In a mean follow up of 27.7 months (range 3-62 months), four patients were alive without evidence of disease. One patient (Patient C) suffered a retroperitoneal recurrence and died 7.3 months after surgery due to chemotherapy-related toxicity.

\section{Discussion}

Tumor or mucin deposits in an inguinal hernia sac present a therapeutic challenge in patients with peritoneal surface malignancies. Appearance of a new groin hernia or worsening of an existing one, may be the presenting symptom of an intra-peritoneal malignancy. In such cases, inguinal dissection with complete resection of the hernial sac should be carried out as part of the cytoreductive surgery and hyperthermic chemotherapy should be applied to the groin while performing the HIPEC procedure. The dissection of the hernial sac in the groin may be technically difficult, especially in cases of invasive disease adherent to neighboring anatomic structures. Including the inguinal area in the perfusion also poses a technical problem.

Patients with large abdominal wall defects following resection of tumor deposits in the abdominal wall, or those patients with large incisional hernias, repaired by synthetic mesh, may need special attention while repairing the hernia defect. In some cases, entrapment of large quantities of perfusate between the skin and the mesh may occur. In order to avoid entrapment of perfused chemotherapy-containing fluid, we have used successfully synthetic mesh with relatively large pores and have had satisfactory results with this technical approach. However, the situ- ation with groin hernias containing tumor deposits is entirely different, since at the time of the perfusion, the inguinal canal is either obliterated following repair or the circulating chemotherapy would not reach the inguinal canal.

The risk of recurrence is high after CRS+HIPEC [15]. Recurrence in the inguinal canal was shown by Liberale et al [16] describing 3 cases of such recurrence in patients with PSM following treatment with CRS and HIPEC or early post-operative chemotherapy (EPIC). The authors described the inguinal canal as a "sanctuary" for PSM. Recurrence in the inguinal canal was also described by others [17]. However, in all previous reports, inguinal involvement was treated with dissection and excision of involved tissues, without the delivery of hyperthermic chemotherapy to the inguinal canal.

The patients presented in our study, were treated with combined intra-peritoneal and inguinal canal perfusion of hyperthermic chemotherapy after achieving complete cytoreduction. We found a simple technical solution to the problem of heated chemotherapy delivery to the inguinal canal. By the addition of an inguinal outflow catheter, we were able to deliver HIPEC successfully to all parts of the abdomen, pelvis and groin utilizing the closed perfusion technique. There was only one complication related to the groin perfusion, infected seroma requiring percutaneous drainage. During a mean follow up of 27.7 months (range 3-62 months) there was no evidence of disease recurrence in the groin. One patient had an early retroperitoneal recurrence of ovarian cancer.

To the best of our knowledge, there are no other reports in the literature of HIPEC treatment through an inguinal incision with perfusion of the inguinal area. Microscopic malignant nests presumably remain within the inguinal area after CRS, in the same manner they do inside the peritoneal cavity. Therefore, it is logical to include this anatomical region in the regions undergoing perfusion after CRS. Extrapolating the data describing high surgical site infection following groin dissection for melanoma [18] would suggest a potential high risk for surgical site infection following inguinal perfusion. One out of five patients in our series had a non-infectious complication that may be attributed to the additional perfusion to the groin (Patient C, Grade II SSI). Moreover, the impact 
of heated chemotherapy on foreign materials such as mesh used in inguinal hernia repair is yet unknown. In our patient population, there were no hernia recurrences documented after an adequate follow-up period.

In conclusion, based on our limited experience, perfusion of the inguinal canal by adding another outflow catheter during HIPEC, following CRS, is relatively safe and effective. Further research is needed to establish the therapeutic yield of this modification of the "traditional" HIPEC method in reducing groin recurrence.

\section{Competing Interests}

The authors have declared that no competing interest exists.

\section{References}

1. Brücher BL, Piso P, Verwaal V, et al. Peritoneal carcinomatosis: cytoreductive surgery and HIPEC--overview and basics. Cancer Invest. $2012 ; 30(3): 209-224$.

2. Sugarbaker PH. Peritonectomy procedures. Ann Surg, 1995;221(1):29-42.

3. Harmon RL, Sugarbaker PH. Prognostic indicators in peritoneal carcinomatosis from gastrointestinal cancer. Int Semin Surg Oncol, 2005;2(1):3.

4. Elias D, Goéré D, Dumont F, et al. Role of hyperthermic intraoperative peritoneal chemotherapy in the management of peritoneal metastases. Eur J Cancer 2014;50(2):332-40

5. Pelz, JO, Stojadinovic A, Nissan A, Hohenberger W, Esquivel J. Evaluation of a peritoneal surface disease severity score in patients with colon cancer with peritoneal carcinomatosis. J Surg Oncol. 2009;99(1):9-15.

6. Valle M, Garofalo A. Laparoscopic staging of peritoneal surface malignancies. Eur J Surg Oncol. 2006;32(6):625-7.

7. Esquivel J, Sugarbaker PH. Pseudomyxoma peritonei in a hernia sac: analysis of 20 patients in whom mucoid fluid was found during a hernia repair. Eur J Surg Oncol. 2001;27(1):54-8.

8. Ghidirim G, Mishin I, Zastavnitsky G. Pseudomyxoma peritonei presenting with inguinal hernia. Chirurgia (Bucur). 2011;106(4):527-9.

9. Hosaka A, Masaki Y, Yamasaki K, Aoki F, Sugizaki K. Pseudomyxoma peritonei presenting with femoral hernias and peritonitis. J Gastrointest Surg. 2007;11(11):1576-8

10. Cichon P, Drucis K, Kakol M, Jastrzebski T, Polec T. Pseudomyxoma peritonei spread into the right inguinal hernia sac--a case report. Pol Merkur Lekarski. 2013;35(208):217-20.

11. Wang T, Vajpeyi R. Hernia sacs: is histological examination necessary? J Clin Pathol. 2013;66(12):1084-6.

12. Campbell P, Dawson S, Wali J, Kenny B, Whiteside MC. Pseudomyxoma peritonei presenting as inguinal hernia. Ulster Med J. 2009;78(3):189-90.

13. Esquivel, J. et al. Cytoreductive surgery and hyperthermic intraperitoneal chemotherapy in the management of peritoneal surface malignancies of colonic origin: a consensus statement. Society of Surgical Oncology. Ann Surg Oncol. 2007;14(1):128-33.

14. Baratti D, Kusamura S, Deraco M. The Fifth International Workshop on Peritoneal Surface Malignancy (Milan, Italy, December 4-6, 2006): methodology of disease-specific consensus. J Surg Oncol. 2008;98(4):258-62.

15. Stojadinovic A, Nissan A, Eberhardt J, et al. Development of a Bayesian Belief Network Model for personalized prognostic risk assessment in colon carcinomatosis. Am Surg. 2011;77(2):221-30.

16. Liberale G, Elias D, Sideris L, Lasser P, Malka D, Sabourin JC, Pocard M. Inguinal canal as an anatomic sanctuary site of relapse in peritoneal carcinomatosis previously treated with intraperitoneal chemotherapy. J Surg Oncol. 2005;91(1):73-6.

17. Cabanas J, Gomes da Silva R, Goldstein P, Verghese M, Sugarbaker PH. Recurrence of pseudomyxoma peritonei within the inguinal canal. Tumori. 2005;91(6):481-6

18. Beitsch P, Balch C. Operative morbidity and risk factor assessment in melanoma patients undergoing inguinal lymph node dissection. Am J Surg. 1992;164(5):462-5. 